OPEN ACCESS

Edited by:

Luca Antonioli,

University of Pisa, Italy

Reviewed by:

Dapeng Chen,

Dalian Medical University, China Lorenzo Bertani,

University of Pisa, Italy

${ }^{*}$ Correspondence:

Keiichi Tominaga

tominaga@dokkyomed.ac.jp

Specialty section: This article was submitted to Gastrointestinal and Hepatic Pharmacology,

a section of the journal

Frontiers in Pharmacology

Received: 11 July 2020 Accepted: 29 December 2020 Published: 29 January 2021

Citation:

Tominaga $K$, Sugaya $T$, Tanaka $T$, Kanazawa $M$, lijima $M$ and Irisawa $A$ (2021) Thiopurines: Recent Topics and Their Role in the Treatment of Inflammatory Bowel Diseases. Front. Pharmacol. 11:582291. doi: $10.3389 /$ fphar.2020.582291

\section{Thiopurines: Recent Topics and Their Role in the Treatment of Inflammatory Bowel Diseases}

\author{
Keiichi Tominaga*, Takeshi Sugaya, Takanao Tanaka, Mimari Kanazawa, Makoto lijima and \\ Atsushi Irisawa
}

Department of Gastroenterology, Dokkyo Medical University, Tochigi, Japan

Ulcerative colitis (UC) and Crohn's disease (CD) are chronic inflammatory bowel diseases (IBD) of unknown etiology, characterized by repeated relapse and remission. The efficacy of thiopurine in IBD was first reported in the late 1960s. Thiopurines are used to alleviate the symptoms of IBD, especially UC. These drugs have a steroid-sparing potential and are widely used for the purpose of maintaining long-term remission in steroid-dependent cases. Therefore, thiopurines tend to be used long-term, but adverse events that accompany long-term use, such as lymphoproliferative disorders, must be monitored with care. In contrast, thiopurine plays a critical role in controlling the immunogenicity of biologics. Furthermore, although thiopurine is an old drug, new findings, including the prediction of serious adverse events such as severe alopecia and acute advanced leukopenia, by nudix hydrolase 15 gene polymorphism analysis, as well as the possibility of appropriate drug monitoring by detailed analysis of 6-thioguanine nucleotides have been clarified. However, the consequences of thiopurine withdrawal have not been determined and further studies, including randomized controlled trials, are necessary to answer the clinical question regarding the scenarios in which thiopurine withdrawal is possible.

Keywords: inflammatory bowel disease, ulcerative colitis, crohn's disease, thiopurine, pharmacological action, biological agents

\section{INTRODUCTION}

Ulcerative colitis (UC) and Crohn's disease (CD) are chronic inflammatory bowel diseases (IBDs) with unknown etiology, characterized by repeated relapse and remission (Ghouri et al., 2020). Rapid induction and ensured maintenance of remission are the foundations of IBD treatment. In UC, remission maintenance therapy is most commonly based on 5-aminosalicylic acid (5-ASA) monotherapy. Contrarily, in cases where it is difficult to maintain remission with 5-ASA monotherapy, immunomodulators (IMs) are used to attain long-term steroid-free remission after initial induction of remission with steroids (Takahashi et al., 2016; Timmer et al., 2016; Matsuoka et al., 2018). IMs are also important for the treatment of CD, where they are used to maintain long-term steroid-free remission and prevent postoperative recurrence (Takahashi et al., 2016; Timmer et al., 2016; Kakuta et al., 2018a; Matsuoka et al., 2018; Gjuladin-Hellon et al., 2019).

The effectiveness of IMs in treating IBD was first reported in the late 1960s (Brooke et al., 1969). Since then, the efficacies of IMs such as azathioprine (AZA), 6-mercaptopurine (6-MP), 


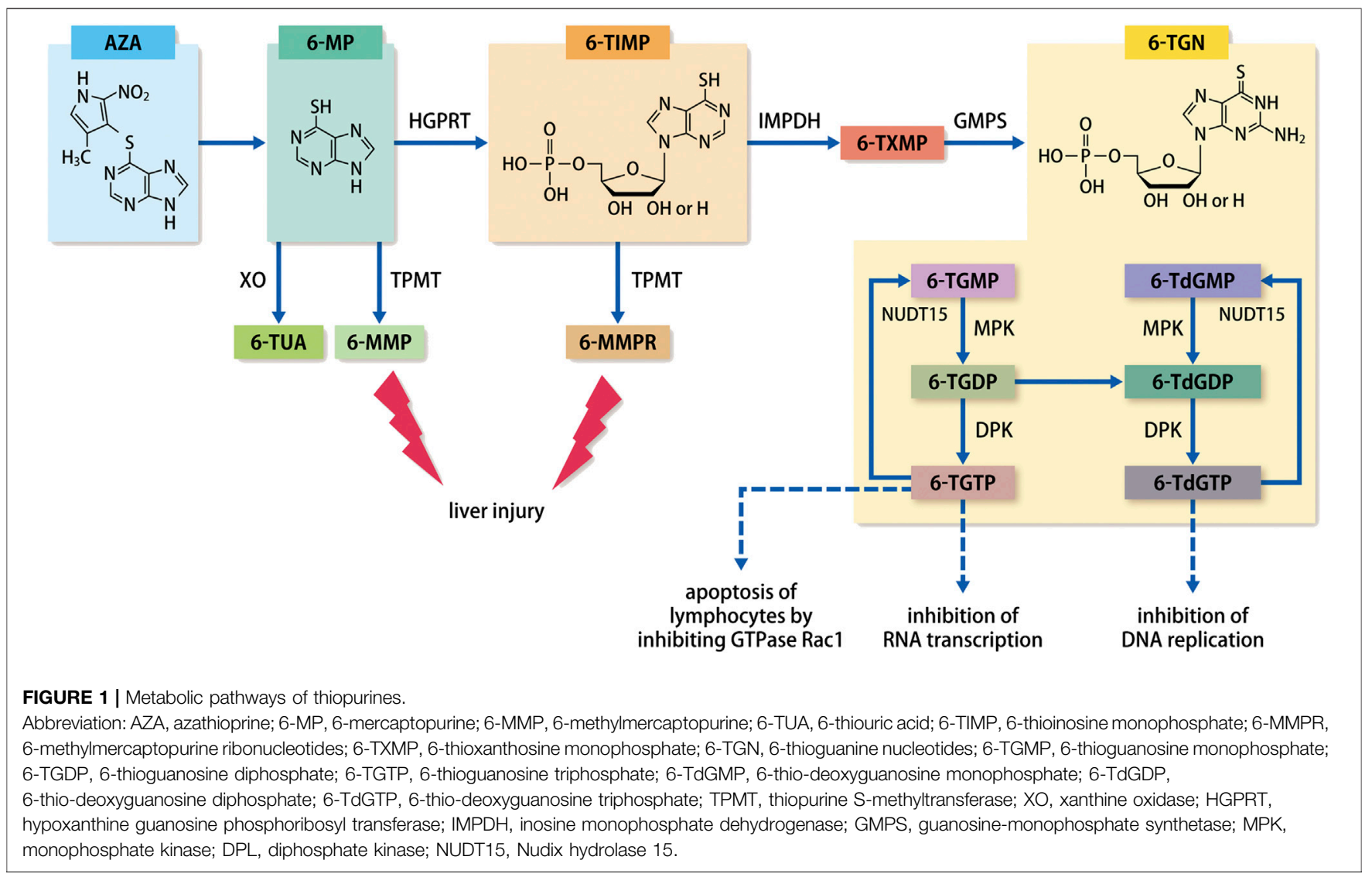

methotrexate, and mycophenolate mofetil have been reported (Smith and Cooper, 2014; Wang et al., 2015). The use of AZA and 6-MP for UC and CD is recommended by the Japanese clinical guidelines (Matsuoka et al., 2018). Herein, we describe the pharmacological actions of thiopurines and their interactions with other drugs. Moreover, we discuss the positioning of thiopurines in the treatment of IBD, as well as the benefits and risks of thiopurine treatment.

\section{Pharmacological Action and Interaction of Thiopurines}

The metabolic pathways of thiopurines are illustrated in Figure 1. AZA is a prodrug that after absorption into the body via the gastrointestinal tract, is promptly converted nonenzymatically to 6-MP which is subsequently metabolically regulated by xanthine oxidase (XO), thiopurine S-methyltransferase (TPMT), and hypoxanthine guanosine phosphoribosyl transferase (HGPRT) (Derijks et al., 2006). Various studies have reported that the combination of $\mathrm{XO}$ inhibitors, allopurinol and thiopurines, increased blood concentrations of 6-thioguanine nucleotides (6-TGN) following its conversion from 6-thioinosine monophosphate (6-TIMP), thereby reducing the dose of thiopurines and enhancing the immunosuppressive effects (Sparrow et al., 2005; Sparrow et al., 2007; Pavlidis et al., 2016). Moreover,
6-MP is converted to 6-methylmercaptopurine (6-MMP) by TPMT; 6-MMP is reportedly associated with an increased risk of liver injury in red blood cells (RBCs) at levels $\geq 5700 \mathrm{pmol} / 8$ $\times 10^{8}$ RBC (Wang and Weinshilboum, 2006; Colleoni et al., 2013). The TPMT gene exhibits a multitude of genetic polymorphisms that affect its enzymatic activity. In particular, more than 30 TPMT gene variants that affect its activity have been reported (Wang and Weinshilboum, 2006; Colleoni et al., 2013). Numerous studies have been performed to address the clinical issues arising from leukopenia in patients with low TPMT activity, as the condition develops due to elevated levels of 6-TGN (Roblin et al., 2011). Polymorphisms in TPMT predominantly result from variant alleles that arise from single nucleotide polymorphisms. Compared with the wild-type TPMT, the TPMT variants are significantly unstable and have lower activity. Based on the assessment of the genotype and TPMT activity, guidelines have been formulated in the US and Europe for determining the initial doses of thiopurines (Relling et al., 2019). However, the activity of TPMT varies with ethnicity. While the standard doses of AZA and 6-MP in the US and Europe are $1.5-2.5 \mathrm{mg} / \mathrm{kg}$ and $1.0-1.5 \mathrm{mg} / \mathrm{kg}$, respectively, the doses in Japan are $1.0 \mathrm{mg} / \mathrm{kg}$ and $0.6 \mathrm{mg} / \mathrm{kg}$, respectively. This is due to the fact that a study confirmed that Asian patients commonly have low TPMT activity (Gomollón et al., 2017; Matsuoka et al., 2018). However, another study reported that the levels of 6-TGN in 
blood are maintained at a therapeutic level in the majority of Japanese IBD patients (Komiyama et al., 2008).

As an active metabolite of thiopurines, 6-TGN ultimately exhibits its pharmacological effects in the form of 6-thio(deoxy)guanosine-triphosphate (6-T(d)GTP). Incorporation of 6-T(d)GTP, which is structurally similar to guanine (a nucleic acid-composing base), into DNA or RNA during chromosome replication or gene transcription leads to the exhibition of its primary role in blocking nucleic acid synthesis and activating lymphocyte proliferation. Previously, blood concentrations of 6TGN were thought to correlate with the effectiveness of IBD treatment, with numerous studies reporting on the utility of monitoring 6-TGN concentrations (Cuffari et al., 1996; Dubinsky et al., 2000; Osterman et al., 2006; Komiyama et al., 2008). Dubinsky reported that a high concentration of $\geq 450 \mathrm{pmol} / 8 \times 10^{8} \mathrm{RBC}$ is linked to a high risk of bone marrow suppression (Dubinsky, 2004). A meta-analysis by Osterman showed that $\geq 230-260 \mathrm{pmol} / 8 \times 10^{8} \mathrm{RBCs}$ correlated substantially with clinical remission (Osterman et al., 2006). Contrarily, 6-TGN concentrations and effectiveness did not correlate strongly, with large variations potentially causing issues, and suggested that non-6-TGN metabolites/ intermediates with immunomodulatory effects may be involved (Lowry et al., 2001; Goldenberg et al., 2004).

\section{NUDT15 Polymorphism}

Questions relating to the 6-TGN concentration and pharmacological effects of thiopurines were later clarified in studies evaluating nudix hydrolase 15 (NUDT15) gene polymorphisms. After thiopurine treatment, Asian patients occasionally present with acute advanced leukopenia combined with advanced alopecia as an adverse event (AE), which is rarely reported in Western populations (Gearry et al., 2004; Palmieri et al., 2007). For this reason, genetic background has been thought to influence Asian-specific thiopurine-related side effects. With recent advances in genome analysis technology, conducting genome-wide correlation analysis has become feasible with relative ease. In 2014, Yang et al. reported that in Korean patients with $\mathrm{CD}$, advanced leukopenia showed an extremely strong correlation with NUDT15 polymorphism (Yang et al., 2014). Several studies on Asian populations soon followed in the fields of blood disorders, neurological disorders, skin disease, and IBD (Asada et al., 2016; Moriyama et al., 2016; Tanaka et al., 2015; Lee et al., 2016; Liang et al., 2016; Zhu et al., 2016; Kim et al., 2017; Shah et al., 2017; Soler et al., 2018; Kishibe et al., 2018. However, all cases were related to NUDT15 codon 139 gene polymorphism, and almost all patients with homozygous risk (Cys/Cys type) presented with severe leukopenia and complete alopecia. An IBD study on Japanese patients revealed that complete alopecia was not only associated with severe leukopenia but was also strongly associated with a NUDT15 polymorphism (Kakuta et al., 2016). NUDT15 polymorphisms occur at a very low frequency in Europeans and Africans (Moriyama et al., 2016). The allele frequency of another NUDT15 variant, p.Gly17_Val18del, is approximately $2 \%$ in Europeans; this variant is also reported to correlate with thiopurine-induced leukopenia (Walker et al., 2019). Nevertheless, none of these reports fully describe the association between complete alopecia and NUDT15 polymorphisms. Given that alopecia is an extremely rare $\mathrm{AE}$ in Caucasians patients taking thiopurines, complete alopecia may be considered a problem specific to Asian populations. In particular, complete alopecia may pose a major problem for young patients, as it can manifest not only as scalp hair loss, but also as entire body hair loss, including eyebrows and armpit hair.

Recently, Kakuta et al. reported a large-scale observational study (MENDEL study) on AEs following the treatment of IBD with thiopurines in Japanese patients (Kakuta et al., 2018b). This Japanese multicenter study analyzed 1,291 Japanese patients with IBD with a history of taking thiopurines and showed that NUDT15 codon 139 gene polymorphism correlated with severe leukopenia and alopecia. This observation led to the inclusion of NUDT15 codon 139 polymorphism detection assays under Japanese national health insurance coverage for thiopurine-naïve patients. Thiopurines are not only used to treat IBD but also many autoimmune diseases, and during immunosuppressive therapy after organ transplantation, the ability to predict whether thiopurines will induce early leukopenia and severe alopecia is welcome news for patients. When treating patients with IBD, it is also recommended that thiopurines are avoided in known carriers of the homozygous risk allele (Cys/Cys) at the NUDT15 codon 139 gene. In heterozygous risk allele (Arg/Cys) carriers, thiopurines are started at approximately half the normal dose, whereas in patients with the wild-type allele (Arg/Arg) thiopurines are started at the normal dose (Kakuta et al., 2020).

Patients with NUDT15 polymorphism may exhibit leukopenia as a dose-dependent AE. Therefore, the concentration of thiopurine metabolite 6-TGN was initially predicted to be elevated. However, Asada et al. reported that 6-TGN levels did not fluctuate in patients with NUDT15 polymorphisms (Asada et al., 2016). A study of pediatric cases of acute lymphoblastic leukemia showed that NUDT15 is an enzyme that dephosphorylates the final active metabolite of thiopurines, 6-T(d)GT, to 6-thio-(deoxy) guanosinemonophosphate (6-T(d)GMP). Moreover, the report showed that coding polymorphisms in NUDT15 suppressed this dephosphorylating function and prevented 6-TGTP and 6TdGTP from being converted to 6-TGMP and 6-TdGMP. This led thiopurine to exhibit excessive pharmacological effects that were manifested principally as lymphocyte apoptosis via Racl inhibition and inhibition of RNA transcription and DNA replication, ultimately causing severe leukopenia (Moriyama et al., 2016). Measurement of 6-TGN concentration includes both 6-T(d)GMP and 6-T(d)GTP; levels of 6-TGN, which had previously been used as an index, do not change with NUDT15 polymorphism. 6-TGTP is incorporated into RNA, and 6-T(d)GTP is incorporated into DNA, where it inhibits RNA transcription and DNA replication, respectively, thereby causing cell apoptosis. 6-TGTP also causes T-lymphocyte apoptosis by inhibiting the activation of GTPase Rac1 (Tiede et al., 2003; Marinković et al., 2014). Therefore, it is suggested that measurement of 6-T(d)GTP concentration is appropriate for efficient drug blood monitoring. 


\section{Effects of Thiopurines in Maintaining Remission}

Prospective data showing the effectiveness of thiopurine monotherapy in inducing remission in UC remains limited. Ardizzone et al. conducted a single-center blinded control study of 5 -ASA at $3.2 \mathrm{~g} /$ day group or AZA at $2 \mathrm{mg} / \mathrm{kg} /$ day group, given the intention of inducing remission in steroiddependent patients with UC. They observed that the remission maintenance rate was $19.4 \%$ in the 5 -ASA group and $52.6 \%$ in the AZA group, demonstrating that AZA had a substantially greater remission rate as well as positive effects in reducing steroids (Ardizzone et al., 2006). However, a considerable amount of time is required for thiopurines to exert their effects (Present et al., 1980), and in actual clinical practice, this agent is infrequently used to induce remission and is more often used in conjunction with steroid reduction in steroid-dependent cases or to maintain remission.

Accumulating evidence from randomized controlled trials (RCTs) and meta-analyses have shown the effects of thiopurines in maintaining remission (Hawthorne et al., 1992; Hibi et al., 2003; Gisbert et al., 2009; Timmer et al., 2016). A comparison between AZA and placebo in the Cochrane Database Systematic Review indicated an odds ratio for remission maintenance failure of $0.41 \quad(95 \%$ confidence interval, 0.24-0.70) (Timmer et al., 2016). A meta-analysis by Gisbert et al. showed that remission maintenance was achieved in $60 \%$ of patients with UC taking thiopurines, but in only $37 \%$ taking the placebo or 5-ASA, demonstrating the benefits of thiopurines (Gisbert et al., 2009).

The efficacy of thiopurines in maintaining remission in patients with $\mathrm{CD}$ has also long been demonstrated. Candy et al. investigated AZA in conjunction with steroid therapy in patients with $\mathrm{CD}$ and reported a markedly higher proportion of patients in remission in the AZA group than in the placebo group after 15 months (Candy et al., 1995).

Recently, powerful immunosuppressive therapies have been introduced to maintain remission, such as anti-tumor necrosis factor-alpha (anti-TNF- $\alpha$ ) antibodies and Janus kinase (JAK) inhibitors. These therapies are used in moderate to severe cases with high disease activity as they can induce and subsequently maintain remission. However, in steroiddependent cases, many guidelines and position statements recommend that an attempt be made to maintain remission using thiopurines prior to these biological agents and JAK inhibitors due to concerns over infection risks and malignancy since many of these expensive drugs are administered via injection (Harbord et al., 2017; Matsuoka et al., 2018; Lamb et al., 2019).

\section{Relationship of Thiopurines With Biological Agents and Small-Molecule Drugs}

By virtue of the introduction of biological agents, clinical treatment for IBD has made considerable progress. At the current height of biological agents, thiopurines are attracting attention for their pharmacological effects for the loss of response
(LOR) (Gisbert and Panés, 2009; Bartelds et al., 2011; van Schouwenburg et al., 2013).

Infliximab (IFX) was the first biological agent introduced to treat IBD; however, as it showed characteristics of a chimeric biological agent due to the approximately $25 \%$ mouse genes, whether this agent should be started as monotherapy or in combination with thiopurines remains contentious. In the SONIC study, the effects of thiopurine monotherapy, IFX monotherapy, and combination therapy of IFX and thiopurines were compared in patients with CD who had not received biological agents or thiopurines (Colombel et al., 2010). This study demonstrated that the clinical remission rate at 26 weeks was substantially greater in the combination therapy group than in the monotherapy group. The appearance ratio of anti-biological antibodies was low in the combination therapy group, and decreased blood concentration of biological agents were confirmed to markedly affect treatment outcomes. The "SUCCESS" RCT, that was similar to the SONIC study, was conducted on 239 patients with moderate to severe UC, who did not receive biologics or thiopurine drugs. The main outcome of that study was the steroid-free remission rate at 16 weeks after starting treatment in the IFX monotherapy, thiopurine monotherapy, and combination therapy groups. The remission rates were $24 \%, 22 \%$, and $40 \%$, respectively, demonstrating that the steroid-free remission rate was markedly greater in the combination therapy group (Panaccione et al., 2014). These results, similar to the aforementioned SONIC study, indicated not only the substantial involvement of thiopurines suppressing the immunogenicity of IFX, but also some effects of thiopurine itself. A meta-analysis that included four articles indicated that the SUCCESS trial, post-hoc analysis of ACT1 and 2, suggested that regardless of previous use of thiopurines, the combination therapy showed a substantially greater remission rate at 4-6 months, although the remission rate at 12 months was not starkly different between combination therapy and monotherapy (Lichtenstein et al., 2009; Christophorou et al., 2015). Presently, the principal biological agents used for treating IBD are human-type biologics and biosimilars. The DIAMOND study is a Japanese RCT which examined the efficacy of thiopurines in combination with a human-type biologic (Matsumoto et al., 2016). This study investigated the efficacy of adalimumab (ADA), a human-type anti-TNFa antibody preparation, in combination with thiopurine, for patients with moderate to severe $C D$ who were naïve to both biologics and thiopurines. However, the DIAMOND study did not elucidate the differences between combination therapy and monotherapy. Although no statistically significant differences were observed in the ADA trough levels and anti-ADA antibody levels, the combination group which was administered ADA and thiopurine, exhibited higher ADA trough levels, and contained a lower proportion of patients positive for anti-ADA antibodies compared to the monotherapy group.

Furthermore, as a different concept from SONIC, SUCCESS, and DIAMOND study, some study has been made of whether the 
TABLE 1 | Adverse event rates for thiopurines in patients with IBD.

\begin{tabular}{|c|c|c|c|c|c|c|c|c|c|}
\hline Ref. & Disease & Case & Leukopenia & Alopecia & $\begin{array}{l}\text { Hepatic } \\
\text { toxicity }\end{array}$ & $\begin{array}{c}\text { Pancreatic } \\
\text { toxicity }\end{array}$ & $\begin{array}{l}\text { Digestive } \\
\text { intolerance }\end{array}$ & Infection & Malignancy \\
\hline Kakuta et al. (2018) & $\mathrm{IBD}$ & 1,282 & $18.2 \%(n=233)$ & $6.8 \%(n=87)$ & $3.7 \%(n=47)$ & $1.7 \%(n=20)$ & $7.2 \%(n=92)$ & $1.3 \%(n=17)$ & $0.2 \%(n=2)$ \\
\hline Calafat et al. (2019) & IBD & 17,365 & $6.5 \%(n=1,134)$ & & $5.1 \%(n=890)$ & $4.2 \%(n=722)$ & $10.3 \%(n=1782)$ & & \\
\hline Pugliese et al., (2018) & UC & 45 & $28.9 \%(n=13)$ & $2.2 \%(n=1)$ & $13.3 \%(n=6)$ & $6.7 \%(n=3)$ & $28.9 \%(n=13)$ & $37.8 \%(n=17)$ & $4.4 \%(n=2)$ \\
\hline Özgenç et al. (2018) & UC & 48 & $2.1 \%(n=1)$ & & & & $6.3 \%(n=3)$ & & \\
\hline
\end{tabular}

IBD, inflammatory bowel disease; Ref., reference; UC, ulcerative colitis.

additional administration of thiopurine restores therapeutic responsiveness when LOR occurs with anti-TNF- $\alpha$ antibody monotherapy. Macaluso et al. reported an analysis of 46 IBD patients treated with anti-TNF- $\alpha$ antibody and additionally treated with thiopurines, methotrexate, and mycophenolate mofetil (Macaluso et al., 2018). Results of this study show that additional combination therapy was effective in 42.4 of CD and $53.8 \%$ of UC. Moreover, the average dose of AZA used at that time was a low dose. These results indicate that the addition of low-dose IM is an effective and safe optimization strategy when LOR occurs with anti-TNF- $\alpha$ antibody monotherapy.

Small-molecule drugs are believed to not elicit anti-drug antibodies, and hence do not induce a LOR via anti-drug antibodies. The JAK inhibitor, tofacitinib, is a small-molecule drug. A report indicated that a stronger therapeutic effect was achieved following the administration of thiopurines in combination with tofacitinib. However, the design of the largescale OCTAVE trial did not allow tofacitinib to be used in combination with thiopurines; hence, the safety of two-agent therapy has yet to be verified. Additionally, the use of thiopurines in combination with tofacitinib has not been recommended due to the risk of infection, particularly viral infection, associated with tofacitinib itself, and concerns that two-agent therapy may induce excessive immunosuppression that could break down the intestinal barrier, thereby making it difficult to control disease activity (Sandborn et al., 2017; Xiong et al., 2019; Feng et al., 2020). Clear evidence is currently lacking on the clinical significance of long-term combination use of biological agents and thiopurines.

\section{Thiopurine-Associated AEs Are Not Linked to NUDT15 Polymorphisms}

Various AEs are often observed with thiopurine treatment. The frequency of AEs in patients with IBD varies from $9 \%$ to $35 \%$, depending on the study (Fraser et al., 2002; Timmer et al., 2016; Kakuta et al., 2018; Gjuladin-Hellon et al., 2019). Table 1 shows the frequency of AEs after thiopurine treatment in IBD (Kakuta et al., 2018; Pugliese et al., 2018; Özgenç et al. 2018; Calafat et al., 2019). Moreover, the appearance of AEs is substantially related to racial differences (Kakuta et al., 2018). AEs are generally categorized into two types: dose-dependent AEs that occur from the pharmacological actions of AZA/6-MP, and dose-independent AEs that are responses to the drug itself. Decreased blood cells, mild alopecia, increased infection susceptibility, and hepatic dysfunction are considered to be dose-dependent AEs, whereas fever, drug eruptions, joint pain, gastrointestinal symptoms, malaise, and acute pancreatitis are considered dose-independent AEs. However, the classification of many AEs is unclear. Gastrointestinal AEs such as nausea, vomiting, and decreased appetite are commonly caused by the compositional binding of the imidazole ring of AZA, and a switch to 6-MP can be beneficial (Kennedy et al., 2013; Calafat et al., 2020). Although conditions can alleviate with time, or proton pump inhibitors and gastrointestinal motilityimproving drugs can be clinically effective, thiopurines must be discontinued if symptoms do not improve even after such treatment.

\section{Involvement of Long-Term Thiopurines and Onset of Lymphoproliferative Disorders (LPDs)}

Thiopurines are commonly used to maintain remission in clinical care for patients with IBD. Consequently, the medication period is inevitably prolonged. Concerns have been raised regarding the increased risk of LPDs such as malignant lymphoma after longterm thiopurine use. According to the CESAMI study, the incidence of LPDs in patients with IBD with no history of taking thiopurines and patients with IBD taking thiopurines were $0.26 / 1,000$ patients/year and $0.90 / 1,000$ patients/year, respectively, indicating an increased risk of thiopurines (Beaugerie et al., 2009). Table 2 presents a summary of several articles describing studies assessing the risks of developing lymphoproliferative disorders when using thiopurines in IBD patients (Beaugerie et al., 2009; Ashworth et al., 2012; Khan et al., 2013; Pasternak et al., 2013; Beigel et al., 2014; Fukata et al., 2014; Wang et al., 2016).

Non-Hodgkin's lymphoma (NHL) comprises the majority of cases of lymphoma in patients with IBD. Moreover, most of these cases test positive for Epstein-Barr virus (EBV). A recent systematic review reported that NHL was the most common histological type $(83.9 \%)$ in the IBD patients. Furthermore, EBV positive status was observed in a large part of lymphoma patients with IBD (44-75\%) (Muller et al., 2020). Patients with IBD who receive thiopurines are likely to develop a subset of lymphoma similar to EBV-related malignant lymphoma that develops after organ transplantation. The prognosis of this subset of lymphoma is poor. Every precaution must be taken for patients who test negative for EBV, especially young male patients, as they are at high risk of developing the aforesaid lymphoma (Kotlyar et al., 2015; Beaugerie et al., 2020). Given the risk of LPDs in patients 
TABLE 2 | Occurrence of lymphoproliferative disorders attributable to thiopurines in patients with IBD.

\begin{tabular}{|c|c|c|c|c|c|c|}
\hline Ref [Ref. No] & Case & TP use & $\begin{array}{c}\text { Previous TP } \\
\text { use }\end{array}$ & $\begin{array}{l}\text { TP never } \\
\text { use }\end{array}$ & TP use (\%) & Disease \\
\hline Beaugerie et al. (2009) & $23 / 19,486$ & $6 / 5,867$ & $2 / 2,809$ & $15 / 10,810$ & & UC, CD, and unclassified IBD \\
\hline Ashworth et al. (2012) & 2/1,374 & 2/839 & & 0/535 & & UC, CD, and unclassified IBD \\
\hline Pasternak et al. (2013) & $95 / 43,969$ & $9 / 5,197$ & & & & UC and CD \\
\hline Khan et al. (2013) & $142 / 45,693$ & $18 / 4,734$ & $5 / 4,662$ & $119 / 36,297$ & & UC \\
\hline Beigel et al. (2014) & $4 / 666$ & 3/262 & $1 / 374$ & $0 / 30$ & & UC and CD \\
\hline Fukata et al. (2014) & $12 / 10,500$ & $2 / 1,341$ & & $10 / 9,159$ & & UC \\
\hline Wang et al. (2016) & $10 / 2,663$ & & & & 8.7 & UC \\
\hline
\end{tabular}

IBD, inflammatory bowel disease; Ref, reference; TP, thiopurines; UC, ulcerative colitis; $C D$, Crohn's disease.

with IBD who receive thiopurines, it is important to examine them for EBV infection, regardless of whether the results are positive or negative.

Long-term use of thiopurines represents a substantial risk factor for the onset of LPD, but this risk is thought to decrease when thiopurines are discontinued, depending on the primary disease condition. However, UC relapse is obviously a concern with such discontinuation or withdrawal. According to MorenoRincón et al., in a retrospective cohort study examining a median sustained remission period of 33 months in patients with UC not on steroid treatment, relapse was observed after thiopurine withdrawal in $32.4 \%$ of patients, and the cumulative relapse rate was $18.9 \%$ at 12 months, $36.5 \%$ at 36 months, and $43.0 \%$ at 60 months (Moreno-Rincón et al., 2015). Similarly, Cassinotti et al. conducted a long-term survey in 155 patients with UC who discontinued AZA during the remission period and found relapse in one-third of patients after 1 year and in half of the patients after 2 years (Cassinotti et al., 2009). We have also previously demonstrated that the relapse rate is greater in the withdrawal group when comparing remission maintenance rates between thiopurine continuation and withdrawal groups of patients with UC with long-term sustained remission, and showed that endoscopic remission (Mayo endoscopic subscore 0) did not guarantee the absence of relapse after withdrawal (Takenaka et al., 2019). As described, a high relapse risk clearly exists with the withdrawal of thiopurines administered as remission maintenance therapy. No clear conclusion has yet been reached on the appropriate and optimal timing of withdrawal, especially considering the concerns for various complications including LPDs after long-term treatment. Nonetheless, due to the various AEs described above, it is necessary to consider thiopurine withdrawal in accordance with disease conditions. Furthermore, thiopurine withdrawal needs to be justified based on assessment of remission with new imaging-enhanced endoscopy or molecular biological examination of tissue samples. Kjærgaard et al. used mucosal biopsy specimens from patients with quiescent UC to analyze mucosal healing in quiescent UC at the molecular and functional level, and reported upregulations of tight junction proteins claudin-2 and claudin-4 mRNA levels, and downregulation of cyclooxygenase-1 enzyme mRNA levels (Kjærgaard et al., 2020). The use of tissue samples to explore mucosal molecular signatures may aid the assessment of remission and could potentially help in deciding when to temporarily withhold thiopurine treatment.

\section{Use of Thiopurines in IBD Patients During the Coronavirus Disease 2019 (COVID-19) Pandemic}

A case of severe acute respiratory syndrome coronavirus 2 (SARS-CoV-2) infection was first reported in December 2019 (Zhu et al., 2020). SARS-CoV-2 is the causative agent of the global COVID-19 pandemic in 2020. Older adults; people with underlying diseases, including diabetes and cardiovascular disease; and patients with cancer are at a high risk of COVID19. These patients are likely to develop severe symptoms and have a high mortality rate (Liang et al., 2020; Xie et al., 2020; Tian et al., 2021). Therefore, there is an increasing concern among health care professionals regarding the effect of immunosuppressive therapy on patients with IBD who receive corticosteroids, thiopurines, or biological agents. However, there is no evidence to indicate that the suffering from IBD increases the risk of contracting COVID-19. Practice guidelines from organizations such as the International Organization of IBD, the European Crohn's and Colitis Organization, the British Society of Gastroenterology, and the American Gastroenterological Association recommend that treatment be continued for IBD patients in remission, even during the COVID-19 pandemic, so that remission can be maintained (Dotan et al., 2020; Kennedy et al., 2020; Rubin et al., 2020). These guidelines state that the discontinuation of immunosuppressive therapy for such patients for the purpose of preventing SARS-CoV-2 infections may lead to the use of higher doses of corticosteroids in the case of relapse of IBD, which may expose the patients to a higher risk than that otherwise (Dotan et al., 2020; Kennedy et al., 2020; Rubin et al., 2020). In particular, there is a dearth of evidence regarding the risk of worsening of respiratory infection in patients receiving thiopurines. However, administration of thiopurine drugs is associated with an increased risk of opportunistic viral infections (Seksik et al., 2009; Harbord et al., 2017; Kirchgesner et al., 2018). Mercaptopurine has been shown to inhibit a protease that is essential for the maturation of Middle Eastern Respiratory Syndrome Coronavirus. However, there are no animal-based models that suggest the clinical efficacy of 
mercaptopurine (Cheng et al., 2015). Based on this finding, many studies have suggested that it is not necessary to discontinue thiopurines in stable patients (Dotan et al., 2020; Kennedy et al., 2020; Rubin et al., 2020).

Currently, there is no evidence based on the ethnicity, age, gender, or treatment to indicate the risks of exacerbation and mortality rates in patients with IBD who have contracted COVID-19. The Surveillance Epidemiology of Coronavirus Under Research Exclusion (SECURE-IBD) is an international database for monitoring and reporting the outcomes of COVID19 in patients with IBD. With the aim of addressing the aforementioned issues, data on cases of IBD patients with COVID-19 are being accumulated in SECURE-IBD for use in various epidemiological studies and therapeutic investigations. Preliminary analyses based on the data obtained from SECUREIBD have led to recommendations that the administration of immunosuppressants be refrained if a patient with IBD who is receiving immunosuppressant therapy, including thiopurines, contracts COVID-19. The treatment should be ceased until the patient recovers from the infection, owing to the high risk of worsened infections (Allez et al., 2020; Brenner et al., 2020). However, as the background of the patients was not examined in detail in these studies, further investigations are necessary in this regard.

\section{CONCLUSION}

In this review, we described the effectiveness, side effects, and challenges of thiopurines in IBD. This review also discussed the use of thiopurine drugs during the COVID-19 pandemic. Thiopurines have been used for many years; however, with the emergence of anti-cytokine therapy in the IBD field, the

\section{REFERENCES}

Allez, M., Fleshner, P., Gearry, R., Lakatos, P. L., and Rubin, D. T. (2020). Care of the patient with IBD requiring hospitalization during the COVID-19 pandemic. J. Crohns Colitis 21, S774-S779. doi:10.1093/ecco-jcc/jjaa150

Ardizzone, S., Maconi, G., Russo, A., Imbesi, V., Colombo, E., and Bianchi Porro, G. (2006). Randomised controlled trial of azathioprine and 5-aminosalicylic acid for treatment of steroid dependent ulcerative colitis. Gut. 55, 47-53. doi:10. 1136/gut.2005.068809

Asada, A., Nishida, A., Shioya, M., Imaeda, H., Inatomi, O., Bamba, S., et al. (2016). NUDT15 R139C-related thiopurine leukocytopenia is mediated by 6-thioguanine nucleotide-independent mechanism in Japanese patients with inflammatory bowel disease. J. Gastroenterol. 51, 22-29. doi:10.1007/s00535-015-1142-4

Ashworth, L. A., Billett, A., Mitchell, P., Nuti, F., Siegel, C., and Bousvaros, A. (2012). Lymphoma risk in children and young adults with inflammatory bowel disease: analysis of a large single-center cohort. Inflamm. Bowel Dis. 18, 838-843. doi:10.1002/ibd.21844

Bartelds, G. M., Krieckaert, C. L., Nurmohamed, M. T., van Schouwenburg, P. A., Lems, W. F., Twisk, J. W., et al. (2011). Development of antidrug antibodies against adalimumab and association with disease activity and treatment failure during longterm follow-up. J. Am. Med. Assoc. 305, 1460-1468. doi:10.1001/jama.2011.406

Beaugerie, L., Brousse, N., Bouvier, A. M., Colombel, J. F., Lémann, M., Cosnes, J., et al. (2009). Lymphoproliferative disorders in patients receiving thiopurines for inflammatory bowel disease: a prospective observational cohort study. Lancet 374, 1617-1625. doi:10.1016/S0140-6736(09)61302-7 importance of thiopurines has been revisited. However, there are no practical guidelines for clinicians regarding the use of thiopurines. An increasing number of analyses on TPMT and NUDT15 have elucidated the pharmacological diversity of drugs for which the metabolism is altered by the genetic makeup of an individual. The use of thiopurines as a key anchor drug for the treatment of IBD is expected to continue. Therefore, future research is necessary for providing evidence regarding the benefit-risk balance in cases where thiopurines are used in combination with a new drug and where the use of thiopurines is withdrawn from patients who have been receiving the drugs for a long period. The formulation of practical guidelines at a global level, by considering the ethnic and genetic background, is necessary for improved personalized medicine. It is also essential to establish evidence regarding the risk of infection, risk of exacerbation, suitability of treatment continuation, and drug interactions with concomitant medications, which would serve as a reference during disease pandemics, including COVID-19.

\section{AUTHOR CONTRIBUTIONS}

Conceptualization: KT. Writing the original draft preparation: KT. Writing review and editing: KT, TS, TT, and MK Supervision: MI and AI.

\section{ACKNOWLEDGMENTS}

We thank Ishida M, Iizuka S, and Nakagawa H (Department of Gastroenterology, Dokkyo Medical University) for technical assistance.

Beaugerie, L., Rahier, J. F., and Kirchgesner, J. (2020). Predicting, preventing, and managing treatment-related complications in patients with inflammatory bowel diseases. Clin. Gastroenterol. Hepatol. 18, 1324-1335. doi:10.1016/j. cgh.2020.02.009

Beigel, F., Steinborn, A., Schnitzler, F., Tillack, C., Breiteneicher, S., John, J. M., et al. (2014). Risk of malignancies in patients with inflammatory bowel disease treated with thiopurines or anti-TNF alpha antibodies. Pharmacoepidemiol. Drug Saf. 23, 735-744. doi:10.1002/pds.3621

Brenner, E. J., Ungaro, R. C., Gearry, R. B., Kaplan, G. G., Kissous-Hunt, M., Lewis, J. D., et al. (2020). Corticosteroids, but not TNF antagonists, are associated with adverse COVID-19 outcomes in patients with inflammatory bowel diseases: results from an international registry. Gastroenterology 159, 481-491.e3. doi:10. 1053/j.gastro.2020.05.032

Brooke, B. N., Hoffmann, D. C., and Swarbrick, E. T. (1969). Azathioprine for Crohn's disease. Lancet 294, 612-614. doi:10.1016/s0140-6736(69) 90325-0

Calafat, M., Mañosa, M., Cañete, F., Ricart, E., Iglesias, E., Calvo, M., et al. (2019). Increased risk of thiopurine-related adverse events in elderly patients with IBD. Aliment. Pharmacol. Ther. 50, 780-788. doi:10.1111/apt.15458

Calafat, M., Mañosa, M., Mesonero, F., Guardiola, J., Mínguez, M., Nos, P., et al. (2020). Switching to a second thiopurine in adult and elderly patients with inflammatory bowel disease: a nationwide study from the ENEIDA registry. J Crohns Colitis. 14, 1290-1298. doi:10.1093/ecco-jcc/jjaa055

Candy, S., Wright, J., Gerber, M., Adams, G., Gerig, M., and Goodman, R. (1995). A controlled double blind study of azathioprine in the management of Crohn's disease. Gut. 37, 674-678. doi:10.1136/gut.37.5.674 
Cassinotti, A., Actis, G. C., Duca, P., Massari, A., Colombo, E., Gai, E., et al. (2009). Maintenance treatment with azathioprine in ulcerative colitis: outcome and predictive factors after drug withdrawal. Am. J. Gastroenterol. 104, 2760-2767. doi:10.1038/ajg.2009.410

Cheng, K. W., Cheng, S. C., Chen, W. Y., Lin, M. H., Chuang, S. J., Cheng, I. H., et al. (2015). Thiopurine analogs and mycophenolic acid synergistically inhibit the papain-like protease of Middle East respiratory syndrome coronavirus. Antiviral Res. 115, 9-16. doi:10.1016/j.antiviral.2014.12.011

Christophorou, D., Funakoshi, N., Duny, Y., Valats, J. C., Bismuth, M., Pineton, D., et al. (2015). Systematic review with meta-analysis: infliximab and immunosuppressant therapy vs. infliximab alone for active ulcerative colitis. Aliment. Pharmacol. Ther. 41, 603-612. doi:10.1111/apt.13102

Colleoni, L., Kapetis, D., Maggi, L., Camera, G., Canioni, E., Cavalcante, P., et al. (2013). A new thiopurine s-methyltransferase haplotype associated with intolerance to azathioprine. J. Clin. Pharmacol. 53, 67-74. doi:10.1177/0091270011435989

Colombel, J. F., Sandborn, W. J., Reinisch, W., Mantzaris, G. J., Kornbluth, A., Rachmilewitz, D., et al. (2010). Infliximab, azathioprine, or combination therapy for Crohn's disease. N. Engl. J. Med. 362, 1383-1395. doi:10.1056/ NEJMoa090449

Cuffari, C., Théorêt, Y., Latour, S., and Seidman, G. (1996). 6-Mercaptopurine metabolism in Crohn's disease: correlation with efficacy and toxicity. Gut. 39, 401-406. doi:10.1136/gut.39.3.401

Derijks, L. J., Gilissen, L. P., Hooymans, P. M., and Hommes, D. W. (2006). Review article: thiopurines in inflammatory bowel disease. Aliment. Pharmacol. Ther. 24, 715-729. doi:10.1111/j.1365-2036.2006.02980.x

Dotan, I., Panaccione, R., Kaplan, G. G., O’Morain, C., Lindsay, J. O., and Abreu, M. T. (2020). Best practice guidance for adult infusion centres during the COVID-19 pandemic: report from the COVID-19 international organization for the study of IBD [IOIBD] task force. J Crohns Colitis. 21, S785-S790. doi:10. 1093/ecco-jcc/jjaa 147

Dubinsky, M. C. (2004). Azathioprine, 6-mercaptopurine in inflammatory bowel disease: pharmacology, efficacy, and safety. Clin. Gastroenterol. Hepatol. 2, 731-743. doi:10.1016/s1542-3565(04)00344-1

Dubinsky, M. C., Lamothe, S., Yang, H. Y., Targan, S. R., Sinnett, D., Théorêt, Y., et al. (2000). Pharmacogenomics and metabolite measurement for 6mercaptopurine therapy in inflammatory bowel disease. Gastroenterology 118, 705-713. doi:10.1016/s0016-5085(00)70140-5

Feng, P. P., Fang, X. S., Zhao, S. H., Fu, J. Y., Zhang, H. T., Yi, Y. L., et al. (2020). Salvianolic acid B decreases interleukin- $1 \beta$-induced colitis recurrence in mice. Chin Med J (Engl). 133 (12), 1436-1444. doi:10.1097/CM9.0000000000000773

Fraser, A. G., Orchard, T. R., and Jewell, D. P. (2002). The efficacy of azathioprine for the treatment of inflammatory bowel disease: a 30 year review. Gut. 50, 485-489. doi:10.1136/gut.50.4.485

Fukata, N., Okazaki, K., Omiya, M., Matsushita, M., and Watanabe, M. (2014). Hematologic malignancies in the Japanese patients with inflammatory bowel disease. J. Gastroenterol. 49, 1299-1306. doi:10.1007/s00535-013-0873-3

Gearry, R. B., Barclay, M. L., Burt, M. J., Collett, J. A., and Chapman, B. A. (2004). Thiopurine drug adverse effects in a population of New Zealand patients with inflammatory bowel disease. Pharmacoepidemiol. Drug Saf. 13, 563-567. doi:10. 1002/pds.926

Ghouri, Y. A., Tahan, V., and Shen, B. (2020). Secondary causes of inflammatory bowel diseases. World J. Gastroenterol. 26, 3998-4017. doi:10.3748/wjg.v26.i28. 3998

Gisbert, J. P., Linares, P. M., McNicholl, A. G., Maté, J., and Gomollón, F. (2009). Meta-analysis: the efficacy of azathioprine and mercaptopurine in ulcerative colitis. Aliment. Pharmacol. Ther. 30, 126-137. doi:10.1111/j.1365-2036.2009. 04023.x

Gisbert, J. P., and Panés, J. (2009). Loss of response and requirement of infliximab dose intensification in Crohn's disease: a review. Am. J. Gastroenterol. 104, 760-767. doi:10.1038/ajg.2008.88

Gjuladin-Hellon, T., Iheozor-Ejiofor, Z., Gordon, M., and Akobeng, A. K. (2019). Azathioprine and 6-mercaptopurine for maintenance of surgically-induced remission in Crohn's disease. Cochrane Database Syst. Rev. 8, CD010233. doi:10.1002/14651858.CD010233.pub3

Goldenberg, B. A., Rawsthorne, P., and Bernstein, C. N. (2004). The utility of 6thioguanine metabolite levels in managing patients with inflammatory bowel disease. Am. J. Gastroenterol. 99, 1744-1748. doi:10.1111/j.1572-0241.2004. 30415.x
Gomollón, F., Dignass, A., Annese, V., Tilg, H., Van Assche, G., Lindsay, J. O., et al. (2017). 3rd european evidence-based consensus on the diagnosis and management of Crohn's disease 2016: Part 1: diagnosis and medical management. J Crohns Colitis. 11, 3-25. doi:10.1093/ecco-jcc/jjw168

Harbord, M., Eliakim, R., Bettenworth, D., Karmiris, K., Katsanos, K., Kopylov, U., et al. (2017). Third european evidence-based consensus on diagnosis and management of ulcerative colitis. Part 2: current management. J Crohns Colitis. 11, 769-784. doi:10.1093/ecco-jcc/jjx009

Hawthorne, A. B., Logan, R. F., Hawkey, C. J., Foster, P. N., Axon, A. T., Swarbrick, E. T., et al. (1992). Randomised controlled trial of azathioprine withdrawal in ulcerative colitis. BMJ 1305, 20-22. doi:10.1136/bmj.305. 6844.20

Hibi, T., Naganuma, M., Kitahora, T., Kinjyo, F., and Shimoyama, T. (2003). Lowdose azathioprine is effective and safe for maintenance of remission in patients with ulcerative colitis. J. Gastroenterol. 38, 740-746. doi:10.1007/s00535-0031139-2

Kakuta, Y., Izumiyama, Y., Okamoto, D., Nakano, T., Ichikawa, R., Naito, T., et al. (2020). High-resolution melt analysis enables simple genotyping of complicated polymorphisms of codon 18 rendering the NUDT15 diplotype. J. Gastroenterol. 55, 67-77. doi:10.1007/s00535-019-01638-x

Kakuta, Y., Kinouchi, Y., and Shimosegawa, T. (2018a). Pharmacogenetics of thiopurines for inflammatory bowel disease in East Asia: prospects for clinical application of NUDT15 genotyping. J. Gastroenterol. 53, 172-180. doi:10.1007/ s00535-017-1416-0

Kakuta, Y., Kawai, Y., Okamoto, D., Takagawa, T., Ikeya, K., Sakuraba, H., et al. (2018b). NUDT15 codon 139 is the best pharmacogenetic marker for predicting thiopurine-induced severe adverse events in Japanese patients with inflammatory bowel disease: a multicenter study. J. Gastroenterol. 53, 1065-1078. doi:10.1007/s00535-018-1486-7

Kakuta, Y., Naito, T., Onodera, M., Kuroha, M., Kimura, T., Shiga, H., et al. (2016). NUDT15 R139C causes thiopurine-induced early severe hair loss and leukopenia in Japanese patients with IBD. Pharmacogenomics J. 16, 280-285. doi:10.1038/tpj.2015.43

Kennedy, N. A., Jones, G. R., Lamb, C. A., Appleby, R., Arnott, I., Beattie, R. M., et al. (2020). British Society of Gastroenterology guidance for management of inflammatory bowel disease during the COVID-19 pandemic. Gut. 69, 984-990. doi:10.1136/gutjnl-2020-321244

Kennedy, N. A., Rhatigan, E., Arnott, I. D., Noble, C. L., Shand, A. G., Satsangi, J., et al. (2013). A trial of mercaptopurine is a safe strategy in patients with inflammatory bowel disease intolerant to azathioprine: an observational study, systematic review and meta-analysis. Aliment. Pharmacol. Ther. 38, 1255-1266. doi:10.1111/apt.12511

Khan, N., Abbas, A. M., Lichtenstein, G. R., Loftus, E. V., Jr., and Bazzano, L. A. (2013). Risk of lymphoma in patients with ulcerative colitis treated with thiopurines: a nationwide retrospective cohort study. Gastroenterology 145, 1007-1015.e3. doi:10.1053/j.gastro.2013.07.035

Kim, S. Y., Shin, J. H., Park, J. S., Kang, S. Y., Nam, T. S., Kim, J. K., et al. (2017). NUDT15 p.R139C variant is common and strongly associated with azathioprine-induced early leukopenia and severe alopecia in Korean patients with various neurological diseases. J. Neurol. Sci. 378, 64-68. doi:10. 1016/j.jns.2017.04.041

Kirchgesner, J., Lemaitre, M., Carrat, F., Zureik, M., Carbonnel, F., and Dray-Spira, R. (2018). Risk of serious and ppportunistic infections associated with treatment of inflammatory bowel diseases. Gastroenterology 155, 337-346.e10. doi:10.1053/j.gastro.2018.04.012

Kishibe, M., Nozaki, H., Fujii, M., Iinuma, S., Ohtsubo, S., Igawa, S., et al. (2018). A. Severe thiopurine-induced leukocytopenia and hair loss in Japanese patients with defective NUDT15 variant: retrospective case-control study. J. Dermatol. 45, 1160-1165. doi:10.1111/1346-8138.14588

Kjærgaard, S., Damm, M. M. B., Chang, J., Riis, L. B., Rasmussen, H. B., HyttingAndreasen, R., et al. (2020). Altered structural expression and enzymatic activity parameters in quiescent ulcerative colitis: are these potential normalization criteria? Int. J. Mol. Sci. 21, 1887. doi:10.3390/ijms21051887

Komiyama, T., Yajima, T., Kubota, R., Iwao, Y., Sakuraba, A., Funakoshi, S., et al. (2008). Lower doses of 6-mercaptopurine/azathioprine bring enough clinical efficacy and therapeutic concentration of erythrocyte 6-mercaptopurine metabolite in Japanese IBD patients. J Crohns Colitis. 2, 315-321. doi:10. 1016/j.crohns.2008.05.002 
Kotlyar, D. S., Lewis, J. D., Beaugerie, L., Tierney, A., Brensinger, C. M., Gisbert, J. P., et al. (2015). Risk of lymphoma in patients with inflammatory bowel disease treated with azathioprine and 6-mercaptopurine: a meta-analysis. Clin. Gastroenterol. Hepatol. 13, 847-858. doi:10.1016/j.cgh.2014.05.015

Lamb, C. A., Kennedy, N. A., Raine, T., Hendy, P. A., Smith, P. J., Limdi, J. K., et al. (2019). British society of gastroenterology consensus guidelines on the management of inflammatory bowel disease in adults. Gut. 68, s1-s106. doi:10.1136/gutjnl-2019-318484

Lee, Y. J., Hwang, E. H., Park, J. H., Shin, J. H., Kang, B., and Kim, S. Y. (2016). NUDT15 variant is the most common variant associated with thiopurineinduced early leukopenia and alopecia in Korean pediatric patients with Crohn's disease. Eur. J. Gastroenterol. Hepatol. 28, 475-478. doi:10.1097/ MEG.0000000000000564

Liang, D. C., Yang, C. P., Liu, H. C., Jaing, T. H., Chen, S. H., Hung, I. J., et al. (2016). NUDT15 gene polymorphism related to mercaptopurine intolerance in Taiwan Chinese children with acute lymphoblastic leukemia. Pharmacogenomics J. 16, 536-539. doi:10.1038/tpj.2015.75

Liang, W., Guan, W., Chen, R., Wang, W., Li, J., Xu, K., et al. (2020). Cancer patients in SARS-CoV-2 infection: a nationwide analysis in China. Lancet Oncol. 21, 335-337. doi:10.1016/S1470-2045(20)30096-6

Lichtenstein, G. R., Diamond, R. H., Wagner, C. L., Fasanmade, A. A., Olson, A. D., Marano, C. W., et al. (2009). Clinical trial: benefits and risks of immunomodulators and maintenance infliximab for IBD-subgroup analyses across four randomized trials. Aliment. Pharmacol. Ther. 30, 210-226. doi:10. 1111/j.1365-2036.2009.04027.x

Lowry, P. W., Franklin, C. L., Weaver, A. L., Pike, M. G., Mays, D. C., Tremaine, W. J., et al. (2001). Measurement of thiopurine methyltransferase activity and azathioprine metabolites in patients with inflammatory bowel disease. Gut. 49, 665-670. doi:10.1136/gut.49.5.665

Macaluso, F. S., Sapienza, C., Ventimiglia, M., Renna, S., Rizzuto, G., Orlando, R., et al. (2018). The addition of an immunosuppressant after loss of response to anti-TNFa monotherapy in inflammatory bowel disease: a 2-year study. Inflamm. Bowel Dis. 18, 394-401. doi:10.1093/ibd/izx010

Marinković, G., Kroon, J., Hoogenboezem, M., Hoeben, K. A., Ruiter, M. S., Kurakula, K., et al. (2014). Inhibition of GTPase Racl in endothelium by 6mercaptopurine results in immunosuppression in nonimmune cells: new target for an old drug. J. Immunol. 192, 4370-4378. doi:10.4049/jimmunol.1302527

Matsumoto, T., Motoya, S., Watanabe, K., Hisamatsu, T., Nakase, H., Yoshimura, N., et al. (2016). Adalimumab monotherapy and a combination with azathioprine for Crohn's disease: a prospective, randomized trial. J Crohns Colitis. 10, 1259-1266. doi:10.1093/ecco-jcc/jjw152

Matsuoka, K., Kobayashi, T., Ueno, F., Matsui, T., Hirai, F., Inoue, N., et al. (2018). Evidence-based clinical practice guidelines for inflammatory bowel disease. J. Gastroenterol. 53, 305-353. doi:10.1007/s00535-018-1439-1

Moreno-Rincón, E., Benítez, J. M., Serrano-Ruiz, F. J., Vázquez-Morón, J. M., Pallarés-Manrique, H., Herrera-Justiniano, J. M., et al. (2015). Prognosis of patients with ulcerative colitis in sustained remission after thiopurines withdrawal. Inflamm. Bowel Dis. 21, 1564-1571. doi:10.1097/MIB. 0000000000000400

Moriyama, T., Nishii, R., Perez-Andreu, V., Yang, W., Klussmann, F. A., Zhao, X., et al. (2016). NUDT15 polymorphisms alter thiopurine metabolism and hematopoietic toxicity. Nat. Genet. 48, 367-373. doi:10.1038/ng.3508

Muller, M., Broséus, J., Feugier, P., Thieblemont, C., Laurent, B., Danese, S., et al. (2020). Characteristics of lymphoma in patients with inflammatory bowel disease: a systematic review. J Crohns Colitis. [Epub ahead of print]. doi:10. 1093/ecco-jcc/jjaa193

Özgenç, F., Karakoyun, M., Ecevit, Ç., Hekimci, H., Kıran Taşçı, E., and Erdemir, G. (2018). Efficacy and safety of long-term thiopurine maintenance treatment for ulcerative colitis in Turkey: a single-center experience. Turk. J. Gastroenterol. 29, 650-654. doi:10.5152/tjg.2018.17151

Osterman, M. T., Kundu, R., Lichtenstein, G. R., and Lewis, J. D. (2006). Association of 6-thioguanine nucleotide levels and inflammatory bowel disease activity: a meta-analysis. Gastroenterology 130, 1047-1053. doi:10. 1053/j.gastro.2006.01.046

Palmieri, O., Latiano, A., Bossa, F., Vecchi, M., D’Incà, R., Guagnozzi, D., et al. (2007). Sequential evaluation of thiopurine methyltransferase, inosine triphosphate pyrophosphatase, and HPRT1 genes polymorphisms to explain thiopurines' toxicity and efficacy. Aliment. Pharmacol. Ther. 26, 737-745. doi:10.1111/j.1365-2036.2007.03421.x

Panaccione, R., Ghosh, S., Middleton, S., Márquez, J. R., Scott, B. B., Flint, L., et al. (2014). Combination therapy with infliximab and azathioprine is superior to monotherapy with either agentin ulcerative colitis. Gastroenterology. 46, 392-400. doi:10.1053/j.gastro.2013.10.052

Pasternak, B., Svanström, H., Schmiegelow, K., Jess, T., and Hviid, A. (2013). Use of azathioprine and the risk of cancer in inflammatory bowel disease. Am. J. Epidemiol. 177, 1296-1305. doi:10.1093/aje/kws375

Pavlidis, P., Stamoulos, P., Abdulrehman, A., Kerr, P., Bull, C., Duley, J., et al. (2016). Long-term safety and efficacy of low-dose azathioprine and allopurinol cotherapy in inflammatory bowel disease: a large observational study. Inflamm. Bowel Dis. 22, 1639-1646. doi:10.1097/MIB.0000000000000827

Present, D. H., Korelitz, B. I., Wisch, N., Glass, J. L., Sachar, D. B., and Pasternack, B. S. (1980). Treatment of Crohn's disease with 6-mercaptopurine. A long-term, randomized, double-blind study. N. Engl. J. Med. 302, 981-987. doi:10.1056/ NEJM198005013021801

Pugliese, D., Aratari, A., Festa, S., Ferraro, P. M., Monterubbianesi, R., Guidi, L., et al. (2018). Sustained clinical efficacy and mucosal healing of thiopurine maintenance treatment in ulcerative colitis: a real-life study. Gastroenterol Res Pract. 2018, 4195968. doi:10.1155/2018/4195968

Relling, M. V., Schwab, M., Whirl-Carrillo, M., Suarez-Kurtz, G., Pui, C. H., Stein, C. M., et al. (2019). Clinical pharmacogenetics implementation consortium guideline for thiopurine dosing based on TPMT and NUDT15 genotypes: 2018 update. Clin. Pharmacol. Ther. 105, 1095-1105. doi:10.1002/cpt.1304

Roblin, X., Oussalah, A., Chevaux, J. B., Sparrow, M., and Peyrin-Biroulet, L. (2011). Use of thiopurine testing in the management of inflammatory bowel diseases in clinical practice: a worldwide survey of experts. Inflamm. Bowel Dis. 17, 2480-2487. doi:10.1002/ibd.21662

Rubin, D. T., Feuerstein, J. D., Wang, A. Y., and Cohen, R. D. (2020). AGA clinical practice update on management of inflammatory bowel disease during the COVID-19 pandemic: expert commentary. Gastroenterology. 159, 350-357. doi:10.1053/j.gastro.2020.04.012

Sandborn, W. J., Su, C., Sands, B. E., D’Haens, G. R., Vermeire, S., Schreiber, S., et al. (2017). Tofacitinib as induction and maintenance therapy for ulcerative colitis. N. Engl. J. Med. 376, 1723-1736. doi:10.1056/NEJMoa1606910

Seksik, P., Cosnes, J., Sokol, H., Nion-Larmurier, I., Gendre, J. P., and Beaugerie, L. (2009). Incidence of benign upper respiratory tract infections, HSV and HPV cutaneous infections in inflammatory bowel disease patients treated with azathioprine. Aliment. Pharmacol. Ther. 29, 1106-1113. doi:10.1111/j.13652036.2009.03973.x

Shah, S. A., Paradkar, M., Desai, D., and Ashavaid, T. F. (2017). Nucleoside diphosphate-linked moiety X-type motif $15 \mathrm{C} 415 \mathrm{~T}$ variant as a predictor for thiopurine-induced toxicity in Indian patients. J. Gastroenterol. Hepatol. 32, 620-624. doi:10.1111/jgh.13494

Smith, M. R., and Cooper, S. C. (2014). Mycophenolate mofetil therapy in the management of inflammatory bowel disease-a retrospective case series and review. J Crohns Colitis. 8, 890-897. doi:10.1016/j.crohns.2014.01.014

Soler, A. M., Olano, N., Méndez, Y., Lopes, A., Silveira, A., Dabezies, A., et al. (2018). TPMT and NUDT15 genes are both related to mercaptopurine intolerance in acute lymphoblastic leukaemia patients from Uruguay. $\mathrm{Br}$. J. Haematol. 181, 252-255. doi:10.1111/bjh.14532

Sparrow, M. P., Hande, S. A., Friedman, S., Cao, D., and Hanauer, S. B. (2007). Effect of allopurinol on clinical outcomes in inflammatory bowel disease nonresponders to azathioprine or 6-mercaptopurine. Clin. Gastroenterol. Hepatol. 5, 209-214. doi:10.1016/j.cgh.2006.11.020

Sparrow, M. P., Hande, S. A., Friedman, S., Lim, W. C., Reddy, S. I., Cao, D., et al. (2005). Allopurinol safely and effectively optimizes tioguanine metabolites in inflammatory bowel disease patients not responding to azathioprine and mercaptopurine. Aliment. Pharmacol. Ther. 22, 441-446. doi:10.1111/j.13652036.2005.02583.x

Takahashi, F., Tominaga, K., Kanamori, A., Takenaka, K., Hoshino, A., Sugaya, T., et al. (2016). Timing for dose-down of 5-ASA depends on mucosal status with ulcerative colitis. Scand. J. Gastroenterol. 51, 827-834. doi:10.3109/00365521. 2016.1141315

Takenaka, K., Tominaga, K., Kanazawa, M., Fukushi, K., Tanaka, T., Kanamori, A., et al. (2019). Endoscopic score vs blood cell indices for determining timing of 
immunomodulator withdrawal in quiescent ulcerative colitis. Sci. Rep. 9, 17751. doi:10.1038/s41598-019-54369-7

Tanaka, Y., Kato, M., Hasegawa, D., Urayama, K. Y., Nakadate, H., Kondoh, K., et al. (2015). Susceptibility to 6-MP toxicity conferred by a NUDT15 variant in Japanese children with acute lymphoblastic leukaemia. Br. J. Haematol. 171, 109-115. doi:10.1111/bjh.13518

Tian, Y., Qiu, X., Wang, C., Zhao, J., Jiang, X., Niu, W., et al. (2021). Cancer associates with risk and severe events of COVID-19: a systematic review and meta-analysis. Int. J. Cancer 15, 363-374. doi:10.1002/ijc.33213

Tiede, I., Fritz, G., Strand, S., Poppe, D., Dvorsky, R., Strand, D., et al. (2003). CD28-dependent Racl activation is the molecular target of azathioprine in primary human CD4+ T lymphocytes. J. Clin. Invest. 111, 1133-1145. doi:10. 1172/JCI16432

Timmer, A., Patton, P. H., Chande, N., McDonald, J. W., and MacDonald, J. K. (2016). Azathioprine and 6-mercaptopurine for maintenance of remission in ulcerative colitis. Cochrane Database Syst. Rev. 18, CD000478. doi:10.1002/ 14651858.CD000478

van Schouwenburg, P. A., Krieckaert, C. L., Rispens, T., Aarden, L., Wolbink, G. J., and Wouters, D. (2013). Long-term measurement of anti-adalimumab using $\mathrm{pH}$-shift-anti-idiotype antigen binding test shows predictive value and transient antibody formation. Ann. Rheum. Dis. 72, 1680-1686. doi:10.1136/ annrheumdis-2012-202407

Walker, G. J., Harrison, J. W., Heap, G. A., Voskuil, M. D., Andersen, V., Anderson, C. A., et al. (2019). Association of genetic variants in NUDT15 with thiopurineinduced myelosuppression in patients with inflammatory bowel disease. J. Am. Med. Assoc. 321, 773-785. doi:10.1001/jama.2019.0709

Wang, L. H., Yang, Y. J., Cheng, W. C., Wang, W. M., Lin, S. H., and Shieh, C. C. (2016). Higher risk for hematological malignancies in inflammatory bowel disease: a nationwide population-based study in Taiwan. Am. J. Gastroenterol. 111, 1313-1319. doi:10.1038/ajg.2016.239

Wang, L., and Weinshilboum, R. (2006). Thiopurine S-methyltransferase pharmacogenetics: insights, challenges and future directions. Oncogene. 25, 1629-1638. doi:10.1038/sj.onc. 1209372
Wang, Y., MacDonald, J. K., Vandermeer, B., Griffiths, A. M., and El-Matary, W. (2015). Methotrexate for maintenance of remission in ulcerative colitis. Cochrane Database Syst. Rev. 11, CD007560. doi:10.1002/14651858.CD007560

Xie, Y., Wang, Z., Liao, H., Marley, G., Wu, D., and Tang, W. (2020). Epidemiologic, clinical, and laboratory findings of the COVID-19 in the current pandemic: systematic review and meta-analysis. BMC Infect. Dis. 20, 640. doi:10.1186/s12879-020-05371-2

Xiong, Y. J., Deng, Z. B., Liu, J. N., Qiu, J. J., Guo, L., Feng, P. P., et al. (2019). Enhancement of epithelial cell autophagy induced by sinensetin alleviates epithelial barrier dysfunction in colitis. Pharmacol. Res. 148, 104461. doi:10.1016/j.phrs

Yang, S. K., Hong, M., Baek, J., Choi, H., Zhao, W., Jung, Y., et al. (2014). A common missense variant in NUDT15 confers susceptibility to thiopurineinduced leukopenia. Nat. Genet. 46, 1017-1020. doi:10.1038/ng.3060

Zhu, N., Zhang, D., Wang, W., Li, X., Yang, B., Song, J., et al. (2020). China novel coronavirus investigating and research team. A novel coronavirus from patients with pneumonia in China, 2019. N. Engl. J. Med. 382, 727-733. doi:10.1056/ NEJMoa2001017

Zhu, X., Wang, X. D., Chao, K., Zhi, M., Zheng, H., Ruan, H. L., et al. (2016). NUDT15 polymorphisms are better than thiopurine S-methyltransferase as predictor of risk for thiopurine-induced leukopenia in Chinese patients with Crohn's disease. Aliment. Pharmacol. Ther. 44, 967-975. doi:10.1111/apt.13796

Conflict of Interest: The authors declare that the research was conducted in the absence of any commercial or financial relationships that could be construed as a potential conflict of interest.

Copyright $\odot 2021$ Tominaga, Sugaya, Tanaka, Kanazawa, Iijima and Irisawa. This is an open-access article distributed under the terms of the Creative Commons Attribution License (CC BY). The use, distribution or reproduction in other forums is permitted, provided the original author(s) and the copyright owner(s) are credited and that the original publication in this journal is cited, in accordance with accepted academic practice. No use, distribution or reproduction is permitted which does not comply with these terms. 